\title{
Synthesizing Evidence for Improving Child Health in India
}

\author{
K SRINATH REDDY AND HENRI VAN DEN HOMBERGH* \\ From Public Health Foundation of India and UNICEF-India*; New Delhi, India. \\ Correspondence to Dr Henri van den Hombergh, Chief-Health, Unicef-India, 73, Lodi Estate, New Delhi, India.
}

$\mathrm{H}$ istorically, research and evidence have played a significant role in designing interventions for improving child health. Research on cholera patients in Bangladesh leading to development of Oral Rehydration Salts (ORS) is one of the most illuminating examples of the contribution of research to a potent public health intervention that has saved many children's lives since then [1]. Similarly, identification of the hand-pump as the source of a cholera outbreak in London led to strategies to the control of one of the most feared scourges in the history of mankind [2]. However, the coverage of many such interventions remains low among populations that most need them. For example, extending the ORS example, only $43 \%$ of children with diarrhea received ORS in the last two weeks in India [3]. There is also a growing apprehension that with increasing integration of programs, the focus on expanding coverage of specific interventions has reduced, leading to dwindling coverage of the known interventions. As a result, decline in child mortality in India remains slow, not sufficient to meet the commitments of Eleventh Five Year Plan or to achieve the Millennium Development Goal.

What then can the research and researchers do? Well, several things. First, synthesize data and present fresh information on the continued presence of the problem. Diarrhea and Pneumonia still kill children in India. Second, synthesize data and present fresh information on what saves lives. Old information becomes dated and stale. Policy needs to be reinfused and programs to be reinvigorated with new data. ORS still save lives. Adding zinc hastens recovery and reduces further episodes. Third, synthesize data and present fresh information on (lack of) progress in expanding coverage of the known interventions. Coverage of ORS and antibiotics for pneumonia remain unacceptably low in India. Fourth, synthesize and present evidence on gaps in the current knowledge to inform the research agenda. We do not even know which organisms cause diarrhea and pneumonia in India. Fifth, synthesize current information on the barriers and enablers for expanding coverage of existing interventions. Children still die due to pneumonia because health facilities are far away and community health workers are not clearly empowered to treat these children [4]. Sixth, synthesize global evidence on what works and what does not? As reported in the paper on review of evidence on acute respiratory infections in this series, management of childhood pneumonia by community health workers in Nepal, some two decades ago, helped reduction of under-five mortality by as much as $28 \%$. [5].

Finally, and most importantly, communicate the evidence to policy makers and program managers in a manner that is clear, actionable and bridges the gap between research and policy. "Community based newborn care by community health workers can reduce the neonatal mortality by as much as $30 \%$, provided the coverage is high, and supervision is intensive" is a simple message, but often not communicated clearly.

However, bridging the gap between evidence and policy is easier said than done! Review of evidence 
on the use of evidence to inform health care management and policy-making in Canada and the United Kingdom provides some lead on how to bridge the gap. Policymakers reported that they would benefit from information that is relevant to decisions highlighted for them and having evidence contextualized to their settings. They further reported that having reviews presented in a way that allows for rapid scanning for relevance and then graded entry are more useful than full reports. One of the ways graded entry can be achieved is through the 1:3:25 principle i.e. 1 page of take home messages, 3 pages of executive summary and 25 pages of report [6]. In another review, policy makers in Australia reported difficulty in accessing useful research syntheses as one of the major barriers in using research findings for policymaking [7]. Equally important is the personal contact between researchers and policymakers. This has been identified as one of the most important facilitators of research use by policymakers, and absence of such a contact becomes a major barrier [8].

This series of systematic reviews, a result of a partnership between Public Health Foundation of India and UNICEF, is an attempt to bridge the gap between evidence and policy for child health programs in India. What is said in this series is often not new, but it clearly provides synthesized evidence on the most effective interventions for improving child survival, identifies critical barriers affecting the scale up of these interventions and lists potential options. It is essential to ensure that the synthesized evidence presented here for the academic and scientific community is communicated in appropriate formats and at appropriate forums to inform the policies and programs. We hope that the exercise will kindle a culture of evidence based programs and policies for improving child health in India, improving not only their design but also their delivery. We invite the academicians and researchers within the child health community to join in generating, synthesizing and communicating relevant evidence for the same.

Competing interests: None stated.

Funding: None.

\section{REFERENCES}

1. Anonymous. 150 years of cholera epidemiology. The Lancet. 2005;366:957.

2. Anonymous. Miracle cure for an old scourge. An interview with Dr Dilip Mahalanabis. Bull WHO. 2009; 87:2.

3. Coverage Evaluation Survey. National Fact Sheet. New Delhi: UNICEF and Ministry of Health and Family Welfare, Govt of India; 2009.

4. Mathew JL, Patwari A, Gupta P, Shah D, Gera T, Gogia S, et al. Acute respiratory infection and pneumonia in India: a systematic review of literature for advocacy and action: UNICEF-PHFI series on newborn and child health, India. Indian Pediatr. 2011;48:191-218.

5. World Health Organization 2000. Handbook of IMCI (Integrated Management of Childhood Illnesses). Available from:http://202.54.104.236/ intranet/eip/immunization manager/pdf/CAH_00_12_Ti_Contents.pdf. Accessed 15 Feb 2011.

6. Lavis J, Davies H, Oxman A, Denis JL, Golden-Biddle K, Ferlie E. Towards systematic reviews that inform health care management and policy-making. Health Serv Res Policy. 2005;10:35-48.

7. Campbell DM, Redman S, Jorm L, Cooke M, Zwi AB, Rychetnic L. Increasing the use of evidence in health policy: practice and views of policy makers and researchers. Aust New Zealand Health Policy. 2009;6:21.

8. Innvaer S, Vist G, Trommald M, Oxman AD. Health policymakers' perceptions of their use of evidence: A systematic review. Health Serv Res Policy. 2002;7:239-44. 